\title{
Activation functions of artificial-neural-network-based nonlinear equalizers for optical nonlinearity compensation
}

\author{
Yuki Miyashita $^{1}$, Takeru Kyono ${ }^{1}$, Kai Ikuta ${ }^{1}$, \\ Yuichiro Kurokawa ${ }^{1}$, and Moriya Nakamura ${ }^{1, \text { a) }}$ \\ ${ }^{1}$ School of Science and Technology, Meiji University, \\ 1-1-1 Higashimita, Tama-ku, Kawasaki, Kanagawa 214-8571, Japan \\ a)m_naka@meiji.ac.jp
}

Abstract: We investigated the performance of artificial neural network (ANN)-based nonlinear equalizers for optical nonlinearity compensation by comparing activation functions, including a sigmoid function, ReLU, and Leaky ReLU. We compared the learning speeds and compensation performances by evaluating the resulting error vector magnitudes of the compensated signals. The performance was investigated using simulated 100-km optical fiber transmission of 10-GSymbol/s 16QAM signals. When the number of hidden-layer units in the ANN was small, the sigmoid function showed better performance in learning speed than ReLU and Leaky ReLU. This point is important because the number of ANN units has to be reduced in order to improve the computational complexity of the ANN-based nonlinear equalizer. Keywords: optical fiber communications, optical nonlinearity, digital signal processing, artificial neural network, activation function

Classification: Fiber-Optic Transmission for Communications

\section{References}

[1] E. Ip, and J.M. Kahn, "Compensation of dispersion and nonlinear impairments using digital backpropagation," J. Lightw. Technol., vol. 26, no. 20, pp. 34163425, Oct. 2008. DOI: 10.1109/JLT.2008.927791

[2] Y. Gao, F. Zhang, L. Dou, Z. Chen, and A. Xu, "Intra-channel nonlinearities mitigation in pseudo-linear coherent QPSK transmission systems via nonlinear electrical equalizer," Opt. Commun., vol. 282, no. 12, pp. 2421-2425, June 2009. DOI: 10.1016/j.optcom.2009.03.002

[3] S. Owaki and M. Nakamura, "Equalization of optical nonlinear waveform distortion using neural-network based digital signal processing," Optoelectronics and Communications Conference (OECC2016), WA2-40, July 2016.

[4] M. Nakamura, Y. Fukumoto, S. Owaki, T. Sakamoto, and N. Yamamoto, "Experimental demonstration of SPM compensation using a complex-valued neural network for 40-Gbit/s optical 16QAM signals," IEICE Commun. Express, vol. 8, no. 8, pp. 281-286, Aug. 2019. DOI: 10.1587/comex.2019XBL0043

[5] Y. Kurokawa, T. Kyono, and M. Nakamura, "Polarization tracking and optical nonlinearity compensation using artificial neural networks," Optoelectron- 
ics and Communications Conference (OECC2020), VP58, Oct. 2020. DOI: 10.1109/oecc48412.2020.9273736

[6] J. Estarán, R. Rios-Müller, M.A. Mestre, F. Jorge, H. Mardoyan, A. Konczykowska, J.-Y. Dupuy, and S. Bigo, "Artificial neural networks for linear and non-linear impairment mitigation in high-baudrate IM/DD systems," European Conference on Optical Communications (ECOC2016), M.2.B.2, Sept. 2016.

[7] V. Kamalov, L. Jovanovski, V. Vusirikala, S. Zhang, F. Yaman, K. Nakamura, T. Inoue, E. Mateo, and Y. Inada, "Evolution from 8QAM live traffic to PS 64QAM with neural-network based nonlinearity compensation on $11000 \mathrm{~km}$ open subsea cable," Optical Fiber Communication Conference (OFC2018), Th4D.5, March 2018.

[8] C. Zhang and P.C. Woodland, "DNN speaker adaptation using parameterised sigmoid and ReLU hidden activation functions," The 41st IEEE International Conference on Acoustics, Speech and Signal Processing (ICASSP2016), SPP1.7, March 2016. DOI: 10.1109/icassp.2016.7472689

[9] J. Tompson, A. Jain, Y. LeCun, and C. Bregler, "Joint training of a convolutional network and a graphical model for human pose estimation," Proc. Conference on Neural Information Processing Systems (NIPS2014), vol. 1, pp. 1799-1807, Dec. 2014.

[10] Y.Lecun, Y. Bengio, and G. Hinton, “Deep learning,” Nature, vol. 521, no. 7553, pp. 436-444, May 2016. DOI: 10.1038/nature14539

[11] R. Rios-Müller, J.M. Estrán, and J. Renaudier, "Experimental estimation of optical nonlinear memory channel conditional distribution using deep neural networks," Optical Fiber Communication Conference (OFC2017), W2A.51, March 2017. DOI: 10.1364/ofc.2017.w2a.51

[12] X. Dai, X. Li, and M. Luo, "Employment of multi-layer neural network for channel equalization in 4x50-Gb/s PAM4 signal transmission over 80-km SSMF," Pacific Rim Conference on Lasers and Electro-Optics (CLEO-PR2018), W1I.4, Aug. 2018. DOI: 10.1364/cleopr.2018.w1i.4

[13] Y. Miyashita, T. Kyono, K. Ikuta, Y. Kurokawa, and M. Nakamura, "Activation function of artificial neural networks for optical nonlinearity compensation," 2020 International Conference on Emerging Technologies for Communications (ICETC2020), I1-4, Dec. 2020.

[14] K. He, X. Zhang, S. Ren, and J. Sun, "Delving deep into rectifiers: Surpassing human-level performance on ImageNet classification,” Proc. 2015 IEEE International Conference on Computer Vision (ICCV), pp. 1026-1034, Feb. 2015. DOI: 10.1109/ICCV.2015.123

\section{Introduction}

Nonlinear waveform distortion caused by optical nonlinearities such as self-phase modulation (SPM) and cross-phase modulation (XPM) is one of the factors that limit the transmission distance of long-haul optical fiber communication systems. Some nonlinear compensation methods based on digital signal processing (DSP) have been investigated, including digital back propagation (DBP) and the Volterra series transfer function (VSTF) [1,2]. However, these methods need an enormous amount of calculations, which increases the power consumption and the delay time at the receiver. On the other hand, artificial neural network (ANN)-based nonlinear equalizers are attracting attention because of their lower computational complex- 
ity $[3,4,5,6,7]$. In recent years, the rectified linear unit (ReLU) has often been employed as an activation function of the ANN-unit instead of the conventional sigmoid function in deep neural networks (DNNs) used for, e.g., speech recognition and image recognition $[8,9]$. ReLU has some advantages compared to the sigmoid function, including fewer vanishing gradient problems and efficient computation [10]. Some investigations of ANN-based nonlinear equalizers also employed ReLU as the activation function $[11,12]$. In this paper, we investigate the performance of three-layer ANN-based nonlinear equalizers by comparing the sigmoid function and ReLU as the activation functions. Besides our former study in [13], we have also investigated the performance of Leaky ReLU [14]. We also investigated how the performance depends on the number of hidden-layer units in the three-layer ANN. Learning speed and compensation performance were compared by numerical simulations of 100-km standard single-mode fiber (SSMF) transmission of a single-channel 16-ary quadrature amplitude modulation (16QAM) signal.

\section{Activation functions of ANN-based nonlinear equalizers}

Figure 1(a) shows the construction of a unit in a complex-valued ANN [4]. The complex inner potential, $u$, is described as

$$
u=\sum_{k=1}^{n} w_{k} x_{k}+b,
$$

where $x_{k}$ is the complex input signal, $w_{k}$ is the complex weight, and $b$ is the complex bias. The complex output of the unit, $y$, is expressed as

$$
y=f(\operatorname{Re}[u])+j \times f(\operatorname{Im}[u]),
$$

where $f$ is the activation function of the unit. Figure 1(b) shows an ANN-based nonlinear equalizer composed of a three-layer complex-valued ANN with a feedforward tapped delay line. The weights and biases are updated in the learning process using the error back propagation (EBP) algorithm. The units in the input and output layers have linear activation functions. The units in the hidden layer have nonlinear activation functions. Figures 1(c), (d), and (e) show three nonlinear activation
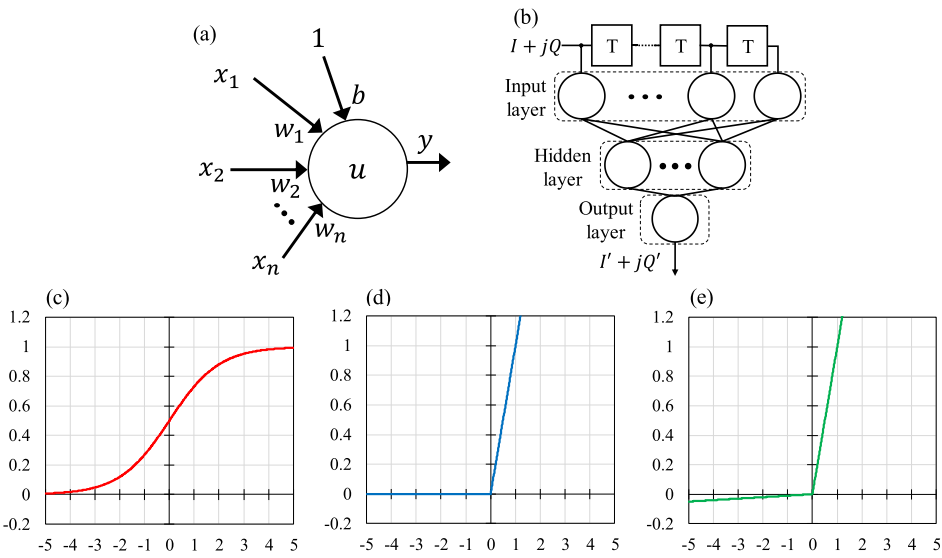

Fig. 1. (a) ANN unit. (b) ANN-based nonlinear equalizer. (c) Sigmoid function. (d) ReLU. (e) Leaky ReLU. 
functions: a sigmoid function, ReLU, and Leaky ReLU, respectively. The sigmoid function has an S-shaped curve that is expressed as

$$
f(x)=\frac{1}{1+e^{-x}} .
$$

One of the advantages of the sigmoid function is that the derivative can be expressed by the output of the function itself as

$$
f^{\prime}(x)=f(x)\{1-f(x)\},
$$

which is used in the EBP algorithm. ReLU and Leaky ReLU are kinds of piecewiselinear functions described as

$$
f(x)=\left\{\begin{array}{cc}
x & (x \geq 0) \\
a x & (x<0),
\end{array}\right.
$$

where $a$ is a coefficient that controls the slope of the negative part of the function. If $a$ is zero, the function becomes a standard ReLU. If $a$ is a small fixed value, typically about 0.01 , the function is called Leaky ReLU [14]. Leaky ReLU can avoid zero gradients over its entire domain, unlike the standard ReLU. This simple calculation contributes to a lower computational cost in both compensation and training processes. However, we can employ a lookup table to reduce the computational cost of the sigmoid function. The comparison of the computational cost of the activation functions depends on the structure of the DSP.

\section{System setup}

Figure 2 shows the optical transmission system used in our simulations. A 10Gsymbol/s 16QAM optical signal was transmitted by 100-km SSMF. The optical fiber had a dispersion of $16.75 \mathrm{ps} / \mathrm{nm} / \mathrm{km}$. The input power to the optical fiber was $10 \mathrm{dBm}$. The noise figure of the Er-doped fiber amplifiers (EDFAs) was 3 $\mathrm{dB}$. The optical signal was received by optical homodyne detection using an optical $90^{\circ}$-hybrid and balanced photodetectors (BPDs). Here, we assumed that the local oscillator (LO) was ideally synchronized to the optical signal. The transmitted signal was distorted by chromatic dispersion and optical nonlinearity. The distorted signal was compensated using the ANN-based nonlinear equalizer in the DSP. We used PRBS $2^{15}-1$ data in the training of the ANN, and PRBS $2^{11}-1$ to evaluate the performance of the system and the equalizer to eliminate the possibility of overfitting. The number of input-layer units was 7 , which is the same as the number of taps of the tapped delay line. The number of hidden-layer units, $N_{\text {hidden }}$, was varied

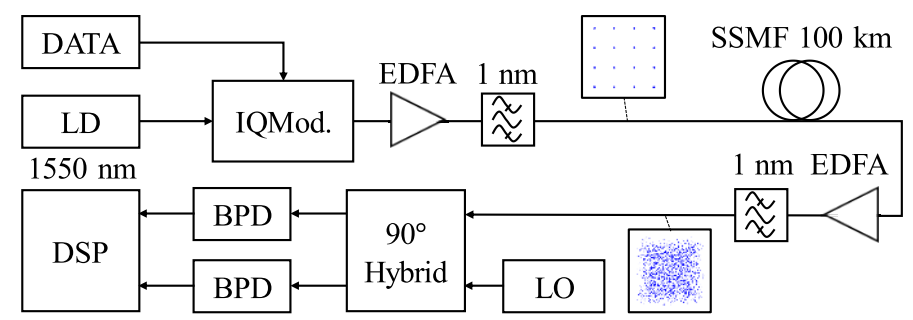

Fig. 2. Setup of 16QAM transmission system. 
from 2 to 20 . The signal quality after the compensation was evaluated by using the error vector magnitude (EVM). Figure 2 also shows the constellations obtained with a back-to-back (BtB) setup and after $100 \mathrm{~km}$ transmission. The EVMs of the constellations were $1.25 \%$ and $43.7 \%$, respectively. The nonlinear equalizer works to reduce the degraded EVM, and the target EVM is $1.25 \%$.

\section{Results and discussion}

Figure 3(a) shows the error versus the number of iterations of the learning steps, where $N_{\text {hidden }}$ was as small as 3 . We performed the learning process 10 times, while changing the random initial values of the weight each time. In this case, ReLU and Leaky ReLU showed large variations in convergence of the learning process. Fig-
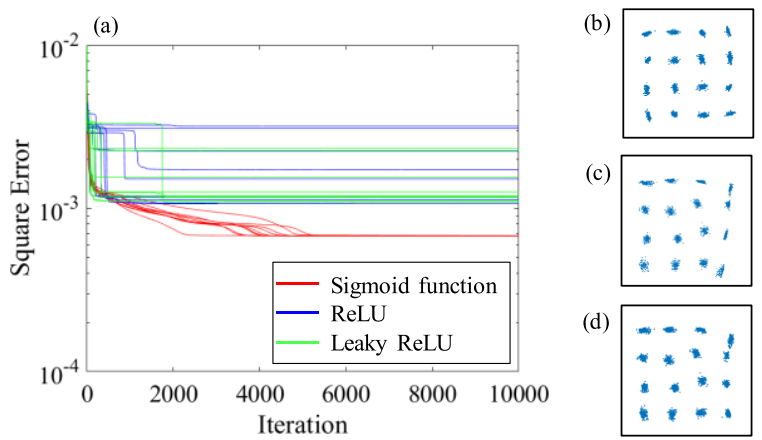

(c)
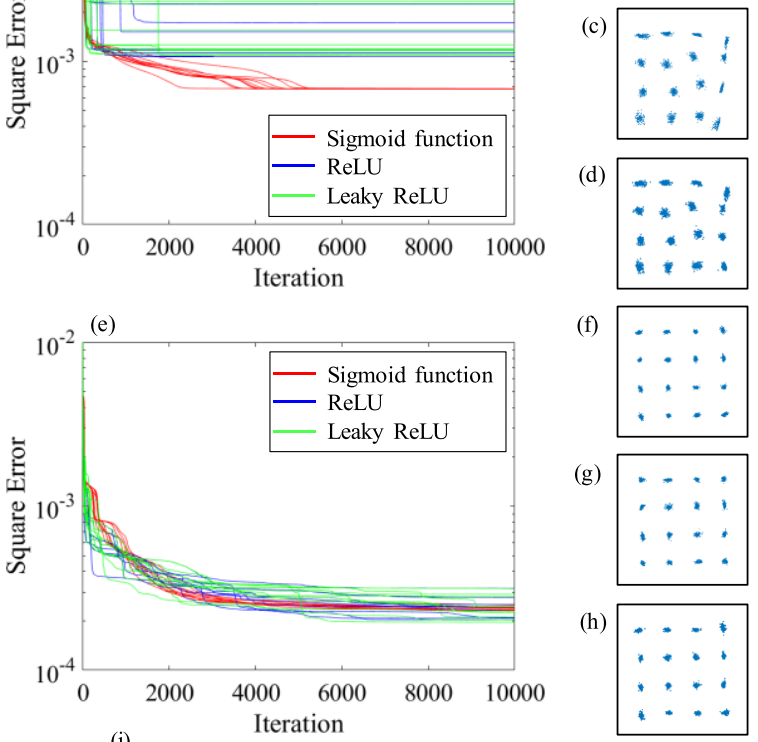

(f)
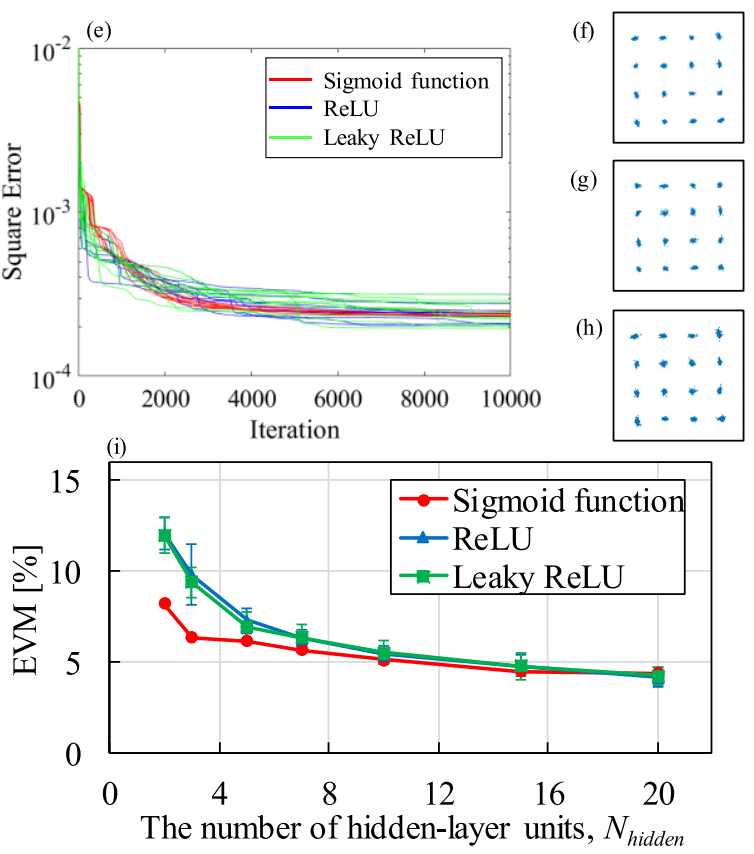

Fig. 3. (a) Error versus iterations of learning steps $\left(N_{\text {hidden }}=\right.$ 3). (b) Constellation (Sigmoid function, EVM = $6.43 \%$ ). (c) Constellation (ReLU, EVM = 12.9\%). (d) Constellation (Leaky ReLU, EVM = 10.9\%). (e) Error versus iterations of learning steps $\left(N_{\text {hidden }}=20\right)$.

(f) Constellation (Sigmoid function, EVM $=4.46 \%$ ). (g) Constellation (ReLU, EVM $=4.95 \%)$. (h) Constellation (Leaky ReLU, EVM $=5.10 \%$ ). (i) EVM versus the number of hidden-layer units. 
ures 3(b), (c), and (d) show the constellations after the nonlinear equalization, where $N_{\text {hidden }}$ was 3, using the sigmoid function, ReLU, and Leaky ReLU, respectively. Each constellation shows the worst case of the compensation results evaluated using the EVM. In the cases of ReLU and Leaky ReLU, we observed serious residual distortion, as shown in Figs. 3(c) and (d). When $N_{\text {hidden }}$ was 20, however, this variation in convergence was improved to some extent, as shown in Fig. 3(e). Figures 3(f), $(\mathrm{g})$, and $(\mathrm{h})$ show the worst constellations after the compensation using the sigmoid function, ReLU, and Leaky ReLU, respectively. When $N_{\text {hidden }}$ was 20, we could not find any significant difference in the compensation performance using the different activation functions. Figure 3(i) shows the EVM after the compensation versus the number of hidden-layer units, $N_{\text {hidden }}$. We plotted the average of the 10 trials of the learning process. The error bars show the standard deviation. When $N_{\text {hidden }}$ was small, the compensation performance using ReLU or Leaky ReLU was degraded in comparison with the case of the sigmoid function. Additionally, EVM showed larger variation. We could not find any significant difference in the compensation performance between ReLU and Leaky ReLU. The simple calculation of ReLU and Leaky ReLU contributes to a lower computational cost. However, we also have to decrease the number of ANN units to lower the computational cost. It is known that ReLU shows good performance in DNNs which include a huge number of hiddenlayer units $[8,9]$. However, it should be noted that ReLU showed different aspects when we used it in the ANN-based nonlinear equalizer with the smaller number of hidden-layer units.

\section{Conclusion}

We investigated the performance of ANN-based nonlinear equalizers for optical nonlinearity compensation, using a sigmoid function, ReLU, and Leaky ReLU as the activation functions of the hidden-layer units. The sigmoid function showed better performance than ReLU and Leaky ReLU when the number of hidden-layer units was small. The sigmoid function requires higher computational cost in both compensation and training processes. One possible way to avoid this problem is to use a lookup table.

\section{Acknowledgments}

This work was supported by JSPS KAKENHI Grant Number JP20K05367 and The Fujikura Foundation. 